\title{
Radiation treatment for refractory endometriosis: a 38-year-old female presenting with vaginal bleeding
}

\author{
Arpan V. Prabhu' ${ }^{1}$, Michael D. Schad ${ }^{2}$, Alexander F. Burnett ${ }^{3}$, Gary D. Lewis ${ }^{1}$ \\ ${ }^{1}$ Department of Radiation Oncology, University of Arkansas for Medical Sciences, UAMS Winthrop P. Rockefeller Cancer Institute, \\ Little Rock, Arkansas, United States \\ ${ }^{2}$ University of Pittsburgh School of Medicine, Pennsylvania, United States \\ ${ }^{3}$ Department of Obstetrics \& Gynecology, University of Arkansas for Medical Sciences, UAMS Winthrop P. Rockefeller Cancer Institute, \\ Little Rock, Arkansas, United States
}

\begin{abstract}
Background: Endometriosis is typically managed with a medical or surgical approach, though some patients have medically refractory disease and are poor surgical candidates.

Case presentation: A 39-year-old woman presented to our facility with uncontrolled bleeding and pain from an endometriotic mass at the vaginal cuff. She had a history of abdominal hysterectomy with bilateral salpingo-oophorectomy, now with medically refractive and inoperable disease due to prior history of vesicovaginal fistula. We prescribed $30 \mathrm{~Gy}$ in 10 fractions with $10 \mathrm{MV}$ and $18 \mathrm{MV}$ photons to the target. At follow-up our patient reported a complete resolution of bleeding and pelvic pain.

Conclusion: Radiation treatment can be an effective treatment for refractory endometriosis.

Key words: endometriosis; radiation therapy; radiotherapy; vaginal bleeding

Rep Pract Oncol Radiother 2021;26(3):457-462
\end{abstract}

\section{Introduction}

Endometriosis is defined as the presence of endometrial-like tissue outside the uterus and is often associated with infertility and significant pain [1], though malignant transformation is rare [2]. It is estimated that up to $10 \%$ of all women of reproductive age are affected by endometriosis [3], though the true prevalence is uncertain as definitive diagnosis requires surgical evaluation. Treatment for endometriosis may be medical, surgical, or both, depending on response and treatment goals. Medical treatment typically involves an oral contraceptive pill (either combined or progestin only) or a gonadotropin-releasing hormone agonist. The surgical approach, which is often considered after failure of hormone therapy, is aimed at complete removal or destruction of endometriotic lesions $[1,4]$. Unfortunately, post-treatment persistence or recurrence of symptoms is not uncommon, regardless of treatment approach $[1,5,6]$.

Literature describing the management of inoperable and medically refractive endometriosis with radiation is limited to a handful of case reports [7-12], and fewer still that report radiation targeted specifically at residual endometriotic tissue. These publications report successful pain and bleeding control $[7,8]$. Here, we present a case of a patient with vaginal bleeding secondary to hormone-refractory and inoperable endometrio- 
sis who was successfully treated with radiation therapy.

\section{Case presentation}

A 39-year-old woman, G0 P0000, with a history of endometriosis and obesity (body mass index 49.6 $\mathrm{kg} / \mathrm{m}^{2}$ ) presented with vaginal bleeding. The patient had a long history of endometriosis for the last 10 years initially treated medically but eventually requiring total abdominal hysterectomy with bilateral salpingo-oophorectomy at an outside facility 8 years prior to the current presentation. She was doing well until 3 years ago when she developed vaginal bleeding with associated pelvic pain. Medical therapy with a gonadotropin-releasing hormone agonist was given for 3 months with no relief in symptoms. After that, an examination under anesthesia showed a $3 \mathrm{~cm}$ nodule at the top of the vagina and biopsy was consistent with endometriosis.

She subsequently underwent robotic resection of that nodule, but her postoperative course was complicated by the development of a vesicovaginal fistula that required surgical correction by urology. Her bleeding temporarily subsided, but returned after 6 months. She was prescribed elagolix (Orlissa), but noted no relief in her symptoms after several months. The gynecologic oncologist did not recommend further surgery given the vesicovaginal fistula requiring surgical repair in the past. Because all conventional treatment options were exhausted, she was referred for possible radiation treatment.

At current presentation, she noted continued intermittent bleeding (requiring 2 pads per day on days of bleeding) and pelvic pain from the endometriosis. Bimanual exam showed a small tender nodularity in the vaginal cuff with a small amount of oozing blood. A discussion was had with the patient to create a plan of action. Given that she had received multiple lines of medical and surgical resection with recurrence of her vaginal bleeding, radiation treatment was offered. The risks, benefits, side effects, and potential complications of radiation treatment, including increased risk of secondary malignancy, were discussed. An MRI of the pelvis was obtained to determine the extent of her endometriosis, and it showed a $3.0 \times 2.3 \times 2.9$ $\mathrm{cm}$ soft tissue mass at the vaginal apex (shown in Fig. 1). The patient was consented for radiation treatment, and a CT simulation was conducted with

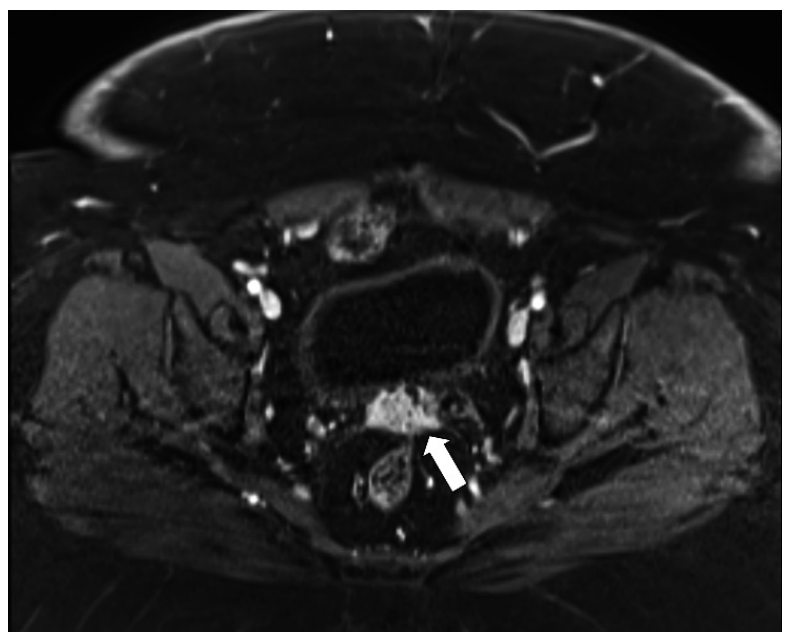

Figure 1. Refractory endometriosis on imaging. Axial T1 post-contrast MRI view showing a $3.0 \times 2.3 \times 2.9 \mathrm{~cm}$ biopsy-proven endometriosis mass at the vaginal apex

both a full and empty bladder. An internal gross target volume (iGTV) was created from contouring and merging GTV volumes from both scans. A clinical target volume (CTV) was not created due to low concern for microscopic disease and this being a benign entity. A uniform $15 \mathrm{~mm}$ expansion in all directions was done to create a planning target volume (PTV). She was prescribed 30 Gy in 10 fractions, and $10 \mathrm{MV}$ and $18 \mathrm{MV}$ photons were used to ensure that $100 \%$ of the prescription dose covered 95\% of the target volume (Fig. 2). Organs at risk (OAR) that were monitored included the bladder, bowel, femurs, and rectum. During weekly checkups, the patient noted improvement in her bleeding with only a small amount of blood with urination.

At follow-up, the patient reported that her vaginal bleeding completely resolved 1 week after radiation treatment completion. She had not had any bleeding since. She also reported resolution of her pelvic pain. She reported this had been the longest period she had had without bleeding in 2 years, and she was very pleased with the results. She denied any lingering side effects and speculum exam showed normal vaginal mucosa and no residual endometriosis tissue at the vaginal cuff.

\section{Discussion}

This report joins a small number of case reports [7-12] that discuss the management of refractory endometriosis with radiation therapy. Most of these case reports describe radiation treatment with the 


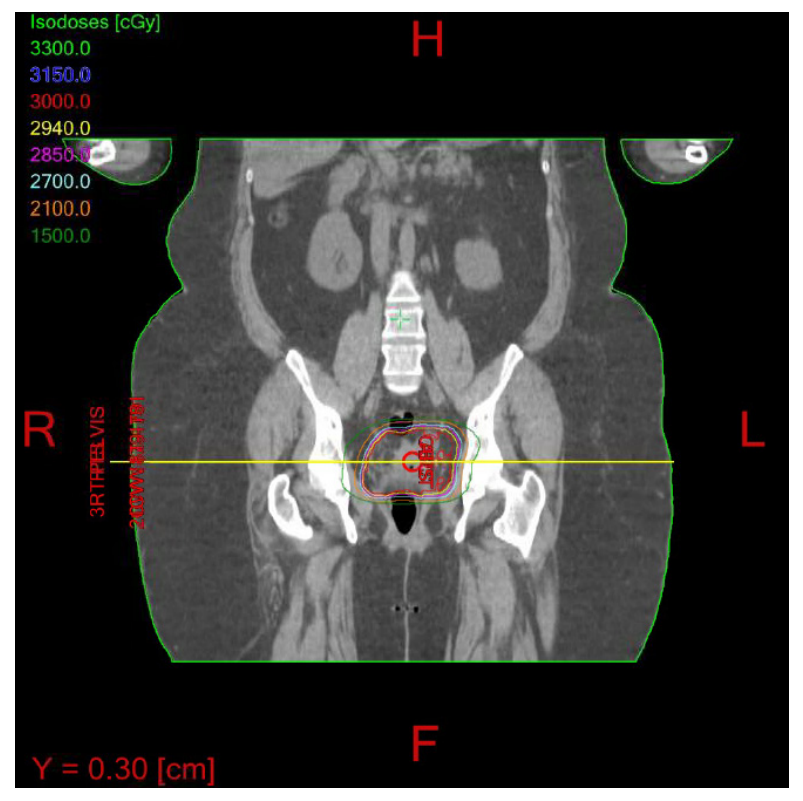

Figure 2. Radiation treatment plan. CT scan coronal view shows isodose lines detailing patient's radiation prescription of $30 \mathrm{~Gy}$ in 10 fractions with $10 \mathrm{MV}$ and $18 \mathrm{MV}$ photons to the target

goal of ovarian ablation $[9,10]$ or destruction of symptomatic ovarian remnants $[11,12]$, as opposed to direct delivery to endometriotic tissue as in this report. Bleeding and pelvic pain were successfully controlled in our patient at follow up, suggesting that radiation may be used to control bleeding in cases where there is no detectable, remaining ovarian tissue.

Our patient was a 39-year-old woman with medically refractive endometriosis who was not a candidate for surgery due to a history of vesicovaginal fistula after a prior surgery. Two other case reports by Kim et al. and Nomiya et al. describe patients with refractory endometriosis treated with radiation targeted to endometriotic tissue $[7,8]$. These patients, 47 and 39 years of age, were deemed to be nonviable surgical candidates due to severe idiopathic thrombocytopenic purpura and exhaustion of surgical options, respectively. Kim et al. reported treatment with 30 Gy in 10 fractions [8], as in our case, while Nomiya et al. administered $20 \mathrm{~Gy}$ in 10 fractions followed by $10 \mathrm{~Gy}$ in 5 fractions for residual bleeding [7]. In both case reports, as well as in our case, bleeding was successfully controlled. As in our patient, Kim et al. also reported resolution of pelvic pain, whereas Nomiya et al. did not describe this outcome.

Management of endometriosis involves either hormonal therapy, surgical removal or destruction of endometriotic lesions, or both, depending on response and treatment goals $[1,4]$. In cases where the goal is pain management and the patient is not currently trying to conceive, an oral contraceptive pill, either combined or progestin only, should be first line. If this treatment fails, it should be followed by a gonadotropin-releasing hormone agonist. Surgery may be considered if both hormone therapies fail. Surgery may be either conservative to the spare ovaries and uterus or definitive with removal of these structures, depending on desire for future conception. Definitive surgery has a lower rate of symptom recurrence. Patients trying to conceive should not be treated with hormonal therapy and are advised to attempt to conceive naturally or to initiate fertility investigations or treatment. Non-steroidal anti-inflammatories, acetaminophen, or opioids may be administered in addition to medical or surgical therapy for pain control $[1,4]$.

Management of endometriosis can be difficult and many patients have persistent or recurrent symptoms, despite medical and surgical management $[1,5,6]$. A randomized controlled trial of 450 women with endometriosis assigned patients to a medical treatment arm with 3 months of a gonadotropin-releasing hormone agonist, a surgical treatment arm with conservative surgery, or a combined arm with both therapies. Recurrence of symptoms of dysmenorrhea, dyspareunia, or abdominal pain at 1 -year follow up was $45 \%$ in the medical-only treatment arm, 50\% in the surgical-only treatment arm, and $40 \%$ in the combined treatment arm [6]. A recent systematic review of treatment outcomes for endometriosis found that the median proportion of patients with no pain reduction, with residual pain, and with a pain recurrence was 11-19\%, 5-59\%, and 17-34\%, respectively [5]. Finally, a recent review article stated approximately half of women treated for endometriosis have recurrent symptoms over a five-year period [1].

In our patient's case, further surgical intervention was not feasible due to her history of vesicovaginal fistula, leading to the consideration of radiation therapy. Radiation has successfully been used in bleeding control for several malignancies including gastrointestinal [13-18], lung [19, 20], and genitourinary tumors $[17,21,22]$. Radiation is also currently used in a number of benign diseases, including hyperproliferative and inflammatory dis- 
ease [23]; however, modern use of radiation for bleeding control of benign diseases is not well reported. Other than the endometriosis case reports previously mentioned, we only identified one other case report of use of radiation for bleeding control in a benign tumor: a 40-year-old man with repetitive vitreous hemorrhage from retinal cavernous hemangioma treated successfully with proton beam therapy to 40 GyRBE in 4 sessions [24]. For our patient, we chose to administer external beam radiation therapy as opposed to brachytherapy. Most studies reporting bleeding control for malignancies as well as the endometriosis case reports utilized external beam radiation as well, with the exception being lung tumors where there was some use of endobronchial brachytherapy for hemoptysis control [20]. Interestingly, a wide range of fractionation and dose schedules of external beam appeared to be equally effective in bleeding control.

There are several potential side effects involved in radiation treatment for endometriosis. Future fertility may be affected by radiation depending on the location of endometriotic tissue and the resultant treatment field. Ovarian failure in premenopausal patients and vaginal stenosis are known to be common gynecological complications of pelvic radiation [25]. Direct DNA damage to ovarian follicles from ionizing radiation leads to follicle death and hastens the natural reduction in follicle number. Low follicle number results in impaired production of ovarian hormones, which leads to inadequate estrogen levels and may result in early menopause [26]. It has also been shown that patients treated with radiation therapy have a low but increased risk of secondary malignancy compared to the general population [27], which is an important consideration in the utilization of radiation therapy for a benign condition. In the case presented here, our patient had previously been treated with a total abdominal hysterectomy with bilateral salpingo-oophorectomy and, therefore, ovarian/fertility preservation was not a concern. We also made the patient aware of the risk of secondary malignancy prior to treatment.

While not common, endometriosis can recur following definitive surgery with total abdominal hysterectomy with bilateral salpingo-oophorectomy [28-30]. This is likely due to incomplete removal of all endometriotic deposits. Additionally, lack of total removal of ovarian tissue can result in ovarian remnant syndrome, where residual ovarian tissue presents as a pelvic mass or pelvic pain [31, 32]. Either of these outcomes can cause significant pain and discomfort for the patient and require additional surgical and medical treatments.

From a historical perspective, radiation has been used successfully for bleeding control in the treatment of endometriosis and other benign gynecological diseases as far back as the early 1900s [33-35]. This practice peaked in the United States and some European countries during the 1930s, 1940s, and 1950s. At the time, abnormal uterine bleeding was thought to be the result of excessive secretion of estrogen relative to progesterone causing anovulatory cycles, and radiation was administered with the intent of either suspending ovarian endocrine function or, for women in their 40s, inducing menopause [33]. This practice, however, fell out of favor due to unnecessary risk of secondary malignancy and discovery of viable medical and surgical alternatives. History therefore serves as a reminder that the use of radiation for benign conditions must be evaluated carefully and that the role of radiation in treating endometriosis should be limited to a select group of patients for whom all other options have been exhausted.

This study has limitations. While all case reports of radiation treatment for treatment-refractory endometriosis reported successful outcomes, publication bias cannot be excluded; patients treated with radiation for endometriosis without adequate symptom control may not have been reported in the literature. Nonetheless, our patient reported the longest period without bleeding in 2 years and resolution of pelvic pain following $30 \mathrm{~Gy}$ in 10 fractions delivered to the endometriotic tissue, and this case joins 2 other case reports also with successful outcomes.

\section{Conclusion}

Vaginal bleeding secondary to refractory endometriosis in the absence of surgical or medical options can be successfully treated with radiation.

\section{Conflicts of interest}

None declared.

\section{Funding}

None declared. 


\section{References}

1. Zondervan $\mathrm{KT}$, Becker $\mathrm{CM}$, Missmer SA. Endometriosis. N Engl J Med. 2020; 382(13): 1244-1256, doi: 10.1056/ NEJMra1810764, indexed in Pubmed: 32212520.

2. Drukała Z, Ciborowska-Zielińska B, Kubrak J, et al. Outcome of a multimodal therapy of a recurrent adenocarcinoma arising from Caesarean section scar endometriosis-A case report. Rep Pract Oncol Radiother. 2010; 15(3): 75-77, doi: 10.1016/j.rpor.2010.03.002, indexed in Pubmed: 24376928.

3. Shafrir AL, Farland LV, Shah DK, et al. Risk for and consequences of endometriosis: A critical epidemiologic review. Best Pract Res Clin Obstet Gynaecol. 2018; 51: 1-15, doi: 10.1016/j.bpobgyn.2018.06.001, indexed in Pubmed: 30017581.

4. Zondervan KT, Becker CM, Koga K, et al. Endometriosis. Nat Rev Dis Primers. 2018; 4(1): 9, doi: 10.1038/s41572018-0008-5, indexed in Pubmed: 30026507.

5. Becker CM, Gattrell WT, Gude K, et al. Reevaluating response and failure of medical treatment of endometriosis: a systematic review. Fertil Steril. 2017; 108(1): 125-136, doi: 10.1016/j.fertnstert.2017.05.004, indexed in Pubmed: 28668150.

6. Alkatout I, Mettler L, Beteta C, et al. Combined surgical and hormone therapy for endometriosis is the most effective treatment: prospective, randomized, controlled trial. J Minim Invasive Gynecol. 2013; 20(4): 473-481, doi: 10.1016/j.jmig.2013.01.019, indexed in Pubmed: 23567095.

7. Nomiya T, Harada M, Sudo H, et al. Radiotherapy for inoperable and refractory endometriosis presenting with massive hemorrhage: a case report. J Med Case Rep. 2012; 6: 308, doi: 10.1186/1752-1947-6-308, indexed in Pubmed: 22989261.

8. Kim KS, Moon WS, Song HW, et al. A case of persistent endometriosis after total hysterectomy with both salpingo-oophorectomy managed by radiation therapy. Arch Gynecol Obstet. 2001; 265(4): 225-227, doi: 10.1007/ s004040000167, indexed in Pubmed: 11789754.

9. Tetar S, Bruynzeel A, Bohoudi O, et al. Pitfalls of Ovarian Ablative Magnetic Resonance-guided Radiation Therapy for Refractory Endometriosis. Cureus. 2018; 10(3): e2294, doi: 10.7759/cureus.2294, indexed in Pubmed: 29750135.

10. Kochbati L, Chaari N, Néji K, et al. [Ovarian irradiation in recurrent endometriosis]. Cancer Radiother. 2005; 9(5): 341-342, doi: 10.1016/j.canrad.2005.06.005, indexed in Pubmed: 16176883.

11. Haglund KE, Viswanathan AN. Computed tomography-based radiation therapy of ovarian remnants for symptomatic persistent endometriosis. Obstet Gynecol. 2008; 111 (2 Pt 2): 579-583, doi: 10.1097/01.AOG.0000299877.71874.91, indexed in Pubmed: 18239029.

12. Thoms W, Hughes L, Rock J. Palliation of recurrent endometriosis with radiotherapeutic ablation of ovarian remnants. Fertil Steril. 1997; 68(5): 938-940, doi: 10.1016/ s0015-0282(97)00342-7, indexed in Pubmed: 9389830.

13. Picardi V, Deodato F, Guido A, et al. Palliative ShortCourse Radiation Therapy in Rectal Cancer: A Phase 2 Study. Int J Radiat Oncol Biol Phys. 2016; 95(4): 1184-1190, doi: 10.1016/j.ijrobp.2016.03.010, indexed in Pubmed: 27215449.
14. Tey J, Choo BAi, Leong CN, et al. Clinical outcome of palliative radiotherapy for locally advanced symptomatic gastric cancer in the modern era. Medicine (Baltimore). 2014; 93(22): e118, doi: 10.1097/MD.0000000000000118, indexed in Pubmed: 25396330.

15. Lee $\mathrm{YH}$, Lee JW, Jang HS, et al. Palliative external beam radiotherapy for the treatment of tumor bleeding in inoperable advanced gastric cancer. BMC Cancer. 2017; 17(1): 541, doi: 10.1186/s12885-017-3508-x, indexed in Pubmed: 28800749.

16. Kawabata, R R, Kameda, C., C, Takata, A., A, et al. [A Case of Unresectable Advanced Gastric Cancer Treated with Palliative Radiation Therapy for Massive Bleeding]. Gan To Kagaku Ryoho. 2017; 44(12): 1653-1655, indexed in Pubmed: 29394732.

17. Sapienza LG, Ning MS, Jhingran A, et al. Short-course palliative radiation therapy leads to excellent bleeding control: A single centre retrospective study. Clin Transl Radiat Oncol. 2019; 14: 40-46, doi: 10.1016/j.ctro.2018.11.007, indexed in Pubmed: 30555940.

18. Kawabata H, Hitomi M, Motoi S. Management of Bleeding from Unresectable Gastric Cancer. Biomedicines. 2019; 7(3): 54, doi: 10.3390/biomedicines7030054, indexed in Pubmed: 31344824.

19. Kramer GW, Gans S, Ullmann E, et al. Hypofractionated external beam radiotherapy as retreatment for symptomatic non-small-cell lung carcinoma: an effective treatment? Int J Radiat Oncol Biol Phys. 2004; 58(5): 1388-1393, doi: 10.1016/j.ijrobp.2003.09.087, indexed in Pubmed: 15050314.

20. Gaito S, Hughes C, Woolf D, et al. Radiotherapy in the control of bleeding from primary and secondary lung tumours. Br J Hosp Med (Lond). 2019; 80(4): 211-215, doi: 10.12968/hmed.2019.80.4.211, indexed in Pubmed: 30951418.

21. Kim DH, Lee JuH, Ki YK, et al. Short-course palliative radiotherapy for uterine cervical cancer. Radiat Oncol J. 2013; 31(4): 216-221, doi: 10.3857/roj.2013.31.4.216, indexed in Pubmed: 24501709.

22. Chen CS, Park S, Shin JiH, et al. Endovascular treatment for the control of active vaginal bleeding from uterine cervical cancer treated with radiotherapy. Acta Radiol. 2018; 59(11): 1336-1342, doi: 10.1177/0284185118758133, indexed in Pubmed: 29448806.

23. McKeown SR, Hatfield P, Prestwich RJD, et al. Radiotherapy for benign disease; assessing the risk of radiation-induced cancer following exposure to intermediate dose radiation. Br J Radiol. 2015; 88(1056): 20150405, doi: 10.1259/ bjr.20150405, indexed in Pubmed: 26462717.

24. Mahdjoubi A, Dendale R, Lumbroso-Le Rouic L, et al. Retinal cavernous haemangioma treated by proton beam therapy. Int Ophthalmol. 2018; 38(2): 759762, doi: 10.1007/s10792-017-0475-9, indexed in Pubmed: 28224302.

25. Viswanathan AN, Lee LJ, Eswara JR, et al. Complications of pelvic radiation in patients treated for gynecologic malignancies. Cancer. 2014; 120(24): 3870-3883, doi: 10.1002/ cncr.28849, indexed in Pubmed: 25056522.

26. Wo JY, Viswanathan AN. Impact of radiotherapy on fertility, pregnancy, and neonatal outcomes in female cancer patients. Int J Radiat Oncol Biol Phys. 2009; 73(5): 1304-1312, doi: 10.1016/j.jijrobp.2008.12.016, indexed in Pubmed: 19306747. 
27. Dracham CB, Shankar A, Madan R. Radiation induced secondary malignancies: a review article. Radiat Oncol J. 2018; 36(2): 85-94, doi: 10.3857/roj.2018.00290, indexed in Pubmed: 29983028.

28. Clayton RD, Hawe JA, Love JC, et al. Recurrent pain after hysterectomy and bilateral salpingo-oophorectomy for endometriosis: evaluation of laparoscopic excision of residual endometriosis. Br J Obstet Gynaecol. 1999; 106(7): 740-744, doi: 10.1111/j.1471-0528.1999.tb08377.x, indexed in Pubmed: 10428534.

29. Namnoum A, Hickman T, Goodman S, et al. Incidence of symptom recurrence after hysterectomy for endometriosis. Fertil Steril. 1995; 64(5): 898-902, doi: 10.1016/s0015-0282(16)57899-6, indexed in Pubmed: 7589631.

30. Shakiba K, Bena JF, McGill KM, et al. Surgical treatment of endometriosis: a 7-year follow-up on the requirement for further surgery. Obstet Gynecol. 2008; $111(6)$ : 12851292, doi: 10.1097/AOG.0b013e3181758ec6, indexed in Pubmed: 18515510.
31. Magtibay PM, Nyholm JL, Hernandez JL, et al. Ovarian remnant syndrome. Am J Obstet Gynecol. 2005; 193(6): 2062-2066, doi: 10.1016/j.ajog.2005.07.067, indexed in Pubmed: 16325616.

32. Kho RM, Abrao MS. Ovarian remnant syndrome: etiology, diagnosis, treatment and impact of endometriosis. Curr Opin Obstet Gynecol. 2012; 24(4): 210-214, doi: 10.1097/ GCO.0b013e3283558539, indexed in Pubmed: 22729094.

33. Sakata R, Kleinerman RA, Mabuchi K, et al. Cancer mortality following radiotherapy for benign gynecologic disorders. Radiat Res. 2012; 178(4): 266-279, doi: 10.1667/ rr2845.1, indexed in Pubmed: 22856888.

34. Brosset $A$. The value of irradiation therapy in the treatment of endometriosis. Acta Obstet Gynecol Scand. 1957; 36(2): 209-225, doi: 10.3109/00016345709157408, indexed in Pubmed: 13434879.

35. Palmer J, Spratt D. Pelvic carcinoma following irradiation for benign gynecological diseases. Am J Obstet Gynecol. 1956; 72(3):497-505, doi: 10.1016/0002-9378(56)90371-4, indexed in Pubmed: 13354658. 